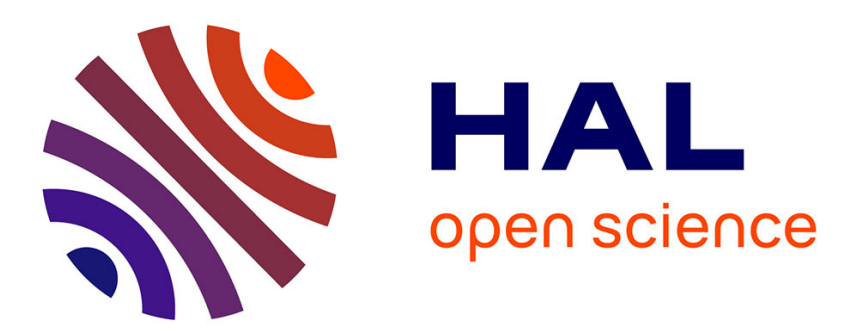

\title{
Wavelength-tunable light shaping with cholesteric liquid crystal microlenses
}

Chloé Bayon, Gonzague Agez, Michel Mitov

\section{To cite this version:}

Chloé Bayon, Gonzague Agez, Michel Mitov. Wavelength-tunable light shaping with cholesteric liquid crystal microlenses. Lab on a Chip, 2014, 14 (12), pp.2063-2071. 10.1039/C4LC00152D . hal01730531

\section{HAL Id: hal-01730531 \\ https://hal.science/hal-01730531}

Submitted on 21 Feb 2022

HAL is a multi-disciplinary open access archive for the deposit and dissemination of scientific research documents, whether they are published or not. The documents may come from teaching and research institutions in France or abroad, or from public or private research centers.
L'archive ouverte pluridisciplinaire HAL, est destinée au dépôt et à la diffusion de documents scientifiques de niveau recherche, publiés ou non, émanant des établissements d'enseignement et de recherche français ou étrangers, des laboratoires publics ou privés. 


\section{Wavelength-tunable light shaping with cholesteric liquid crystal}

\section{microlenses}

\section{Chloé Bayon, Gonzague Agez and Michel Mitov*}

The ability to guide light at the mesoscopic scale is important both scientifically and technologically. Especially relevant is the development of wavelength-tunable light-shaping microdevices. Here we demonstrate the use of cholesteric liquid crystal polygonal textures organized as an array of microlenses for this purpose. The beam shaping is controlled by tuning the wavelength of the incident light in the visible spectrum. By taking advantage of the self-organization property of liquid crystals, the structure of the lens and its optical response are tailored by changing the annealing time of the single layer material during a completely integrated one-step process. The intrinsic helical organization of the layer is the cause of the light shaping, and not the shape of the surface as for conventional lenses. A new concept of light manipulation using the structure chirality of liquid crystals is demonstrated, which concerns soft matter photonic circuits to mould the light.

Article history: Received $4^{\text {th }}$ February 2014 / Accepted 16 $6^{\text {th }}$ March $2014 /$ First published (online): 02 ${ }^{\text {th }}$ May 2014

https://doi.org/10.1039/C4LC00152D

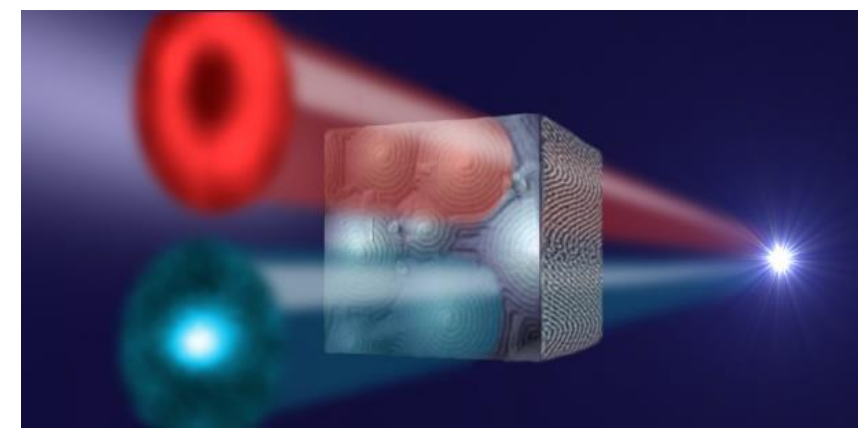

Centre d'Elaboration de Matériaux et d'Etudes Structurales (CEMES), CNRS, 29 rue J. Marvig, 31055 Toulouse cedex 4, France. *Corresponding author, e-mail : mitov@cemes.fr 


\section{Introduction}

\section{Motivation of the research}

The field of optics and photonics deals with controlling the propagation of electromagnetic radiation using mirrors, lenses, fibres and diffractive elements ${ }^{1-9}$. Optical component engineers are currently working towards the miniaturisation (on and off chips) at the micro-and nano-scale. A microlens is a lens with a diameter that typically ranges from 10 to $100 \mu \mathrm{m}^{10}$. In the field of micro-optics and integrated optics ${ }^{11}$, there is a growing interest in parallelizing microlenses to make an array which includes two or more lenses, wherein the individual components are arranged and sized so that there are substantially no gaps between lenses through which incident light can pass. Microlens arrays are widely used in lab-on-a-chip optical devices requiring interconnections (such as fibre coupling, signal injection and/or detection enhancement), storage systems, photovoltaic devices, and to improve the light efficiency of liquid crystal display (LCD) panels for projectors and 3D imaging. Various fabrication methods combined with lithographic techniques have been demonstrated like gray-scale photolithography ${ }^{12}$, holographic lithography, photothermal patterning, inkjet printing ${ }^{13}$, LIGA process, laser ablation ${ }^{14}$, melting photoresist (reflow technique) ${ }^{15}$, or hot embossing methods based on electrohydrodynamic deformations ${ }^{16}$. However these techniques are limited to the case of microlens arrays on flat solid substrates and are time-consuming by requiring multiple steps ${ }^{11}$ like resist coating, baking, wet processing, developing or etching. One-step self-assembly approaches are thus promoted since they provide simple and low-cost methods with large-area patterns.

\section{Scope and aims of the present study}

A key objective in the field of microlenses lies in the tuning of lens properties. Here we demonstrate the wavelength-tunable light shaping properties of cholesteric LC (CLC) microlenses, which can be grown on a variety of substrates by a complete self-assembly process. The resulting lens properties 
are tuned by controlling the annealing time of the LC layer, and used at room temperature (RT) afterwards. The CLC phase spontaneously forms a macroscopic helical structure when either the LC molecules are inherently chiral or when chirality is introduced. The presence of chirality causes the director profile to assume a twisted configuration throughout the medium. Photonic band structures have attracted considerable attention in recent years because of their ability to control the propagation of light over a range of different frequencies ${ }^{17-19}$, and a CLC is regarded as a 1D photonic crystal since the key feature of a CLC is the existence of a bandgap for visible light ${ }^{20}$. Due to its (periodic) helical structure, a CLC may indeed exhibit the basic property of selective light reflection which follows Bragg's law. Here the Bragg structure is a single layer of CLC shaped into an array of polygons (Fig. 1 a). As shown below, the propagation of the transmitted light and the focus properties of the layer are directly attributed to the bulk structure, which is associated to the film's intrinsic helical organization.

This work falls within the framework of liquid-crystal tunable photonic devices that have recently attracted significant research interest as potential candidates for low-cost, highly functional photonic elements $^{21}$. As a solid film that can be coated on different substrates including non-planar (Fig. 1 b), flexible or rollable substrates (Fig. $1 \mathrm{c}$ ), the layer offers the combined benefits of multiple light manipulation capabilities, seamless integration and mechanical stability. The layer thus provides a versatile and single microdevice to be integrated as a part of a lab-on-a-chip optical system. Instead of relying on microscopic flow, as in most optofluidic systems, the optical system is fabricated in a one-step (self-organization) process in a viscous state, and then solidified to become a microlens array that is periodically modulated at the molecular level. This modulation determines the optical properties of the system, which is a Bragg medium and exhibits wavelength tunability. 


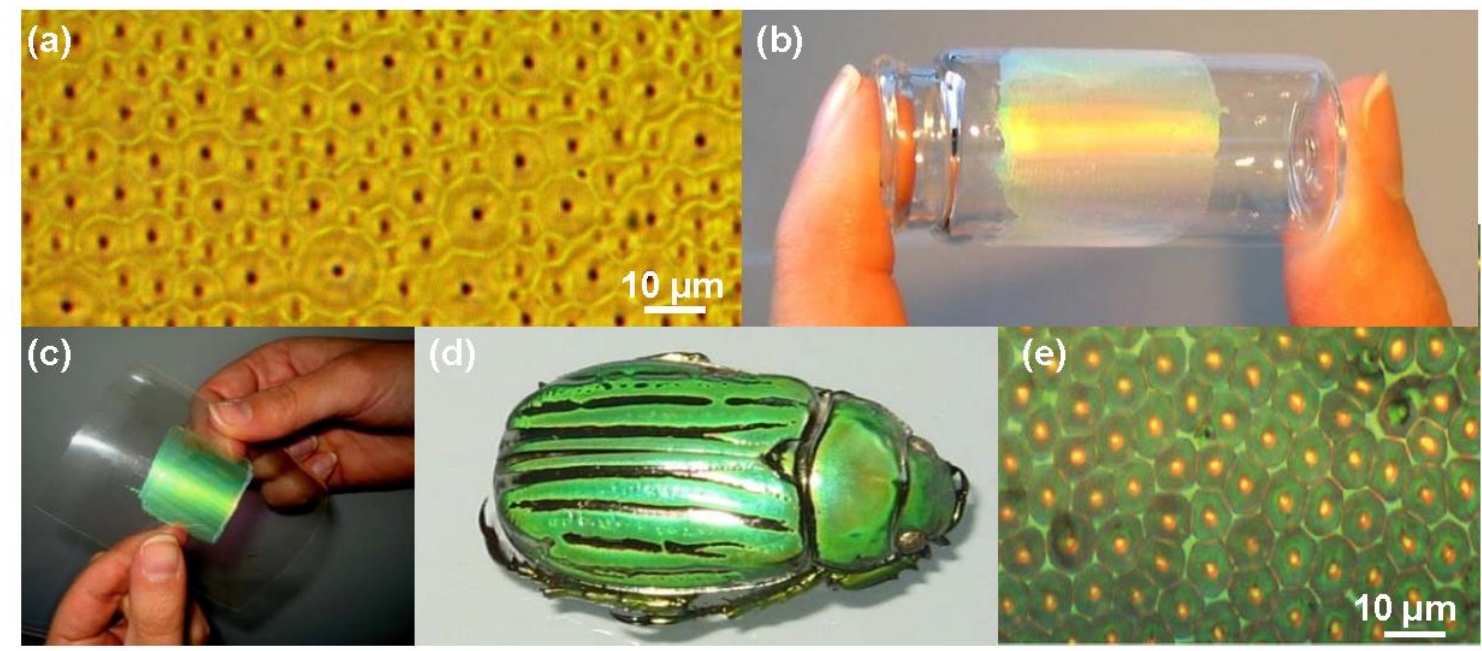

Fig. 1 The optical film. (a) Polygonal microtexture (unpolarised light microscopy, transmission mode). Coating of the film on a: (b) curved solid substrate or (c) on a flexible plastic foil. The film colour depends on the illumination conditions. (d) Analogy with an optical material in living matterthe carapace of Chrysina Gloriosa beetle, with its green and silver stripes. (e) Polygonal microtexture exhibited by the green stripes (unpolarised light microscopy, reflection mode).

\section{Design and working principles}

CLC oligomers from Wacker Chemie $\mathrm{GmbH}$ were used ${ }^{22}$. The molecule consists of a siloxane cyclic chain to which two types of side chains are attached via aliphatic spacers: an achiral mesogen and a chiral cholesterol-bearing mesogen. The molar percentage of chiral mesogen in the molecule can vary from $31 \%$ (in the case of Silicon Red (SR)) to $50 \%$ (in the case of Silicon Blue (SB)). The pitch of the helical structure and therefore the reflection wavelength depend on the SB:SR proportion. We blended SB and SR compounds at 40:60 wt\%, thus the mean position of the reflection band is located in the middle of the visible spectrum $(560 \mathrm{~nm})$. The cholesteric phase appears between $180-210^{\circ} \mathrm{C}$ (clearing temperature range) and $40-50^{\circ} \mathrm{C}$ (glass-transition temperature range). We were easily able to freeze the film into a glassy solid state by simple thermal quenching on a metal plate after 
annealing. Advantageously, the unique properties of the mesomorphic layer can thus be studied at RT.

The oligomer film was confined between two glass plates which were separated by spacers to control the thickness of the film. In our case we used Mylar spacers that were $13 \pm 2 \mu \mathrm{m}$ thick. No peculiar surface treatment or alignment layers were used. The samples were kept at $140^{\circ} \mathrm{C}$ (in the cholesteric phase) for 2 minutes. We performed rapid thermal quenching by depositing the sample on a metallic substrate at RT. The cholesteric film changed from a viscous to a glassy solid state. The samples were kept at a low temperature $\left(2^{\circ} \mathrm{C}\right)$ for 15 minutes in order to remove the cover glass more easily and to obtain a smooth interface. After this, the film is ready to be annealed. The formation of the optical network is extremely simple. The film is kept in a viscous state at $140^{\circ} \mathrm{C}$ for a variable annealing time. The polygonal texture (Fig. 1a) spontaneously nucleates and grows. The material is then quickly quenched. The structure of the layer after annealing is fixed at RT into a solid film. The structure of the layer is the result of a compromise between the surface tension and the cholesteric organization. The surface tension minimizes the surface area by rearranging the fluid interface from a planar state to a polygonal field. The formation of the polygonal texture is driven by the antagonistic character of the surface anchoring-i.e. rod-like liquid-crystalline molecules preferentially align tangentially to the substrate and perpendicularly to the air interface. Inside polygons, the cholesteric texture arranges into double spirals with a pitch related to the helical pitch $^{23}$. Spiral patterns are frequent in CLC textures at equilibrium in synthetic ${ }^{24}$ and biological ${ }^{25}$ materials, when they are subjected to an electric stimulus ${ }^{26,27}$ or doped with gold nanoparticles ${ }^{28}$. Our optical film may be implemented as a thin and lightweight optical network with a remarkable versatility in a large range of applications. It may be coated on: (i) a flat glass substrate (like it is here for fundamental investigations; it could as well be fabricated on a silicon substrate with the aim to associate optical functions with electronic functions); (ii) a curved solid surface (Fig. 1b); (iii) a rollable plastic sheet (Fig. 1c) with curvature radii comparable to those of the cuticle of the Chrysina Gloriosa beetle (Fig. 1d). The chitin-based exoskeleton of this insect includes green stripes with polygons (Fig. 
1e) which are structurally analogous to the polygons formed spontaneously at the free surface of CLC open films ${ }^{23}$. Biological systems exploit a large variety of photonic structures by utilizing periodic architecture on the micro- and nanoscales to achieve various optical functions like microlens arrays ${ }^{29}$; artificial photonic structures mimicking the cuticle of some beetles ${ }^{30}$ and the brittlestar's skeletal ossicles $^{31}$ are typical examples. The question of the biological function of the polygonal texture of Chrysina Gloriosa is still debated. The intrinsic asymmetry of the related cholesteric structure ${ }^{32,33}$ could play a role in the radiative thermoregulation of the beetle (when the energy flow is strongest from the centre of the body towards the outside $\left.{ }^{34}\right)$. The polygonal textures in LCs have been known for some time $e^{24,35}$. Recently, we have shown that the reflection colour of an open CLC film with polygonal textures may be tuned by controlling the annealing time of the film ${ }^{23}$. As evidenced by transmission electron microscopy (TEM), the colour changes are due to distortions in the orientation of the helical axis inside the bulk of the optical layer (Supplementary Fig. S1). These distortions propagate more deeply in the layer thickness as the annealing time increases. The colour depends on the angle between the observation direction and the helical axis. The structure of such cholesteric films is complex since this orientation continuously varies between the air interface and the substrate. The full description of the 3D optical properties of the cholesteric polygonal texture, its action as an optical network of microlenses, and the demonstration of its light shaping properties are not reported in the literature.

\section{Experimental results and discussion}

We used a spectrometer equipped with a standard confocal microscope (Fig. 2) to scan the layer over the area of a single polygon, including the spaces (air) below and above the layer. All the optical measurements were performed at RT. In order to normalize the stacks of spectra (associated with a map), it is necessary to realize a spectrum of transmitted white light through a glass plate without any extra material (transmission baseline). Then we can divide each stack of spectra (i.e. each map) 
by this spectrum. An XY map contains 841 (29x29) pixels so the scanning area of a map is $14.5 \times 14.5$ $\mu \mathrm{m}^{2}$. One $\mathrm{XY}$ transmission map is then related to a collection of 841 transmittance spectra. The longitudinal ( $\mathrm{Z}$ ) resolution of the confocal microscope is $2 \mu \mathrm{m}$, so one pixel of the XY map integrates information inside a voxel (volumetric element) with $0.5 \mu \mathrm{m} \times 0.5 \mu \mathrm{m} \times 2 \mu \mathrm{m}$ dimensions. In order to have a full 3D description, $X Y$ maps were recorded for 27 consecutive $Z$ foci. On this third spatial $Z$ dimension, the scanning distance is $104 \mu \mathrm{m}$ with a Z-step of $4 \mu \mathrm{m}$.

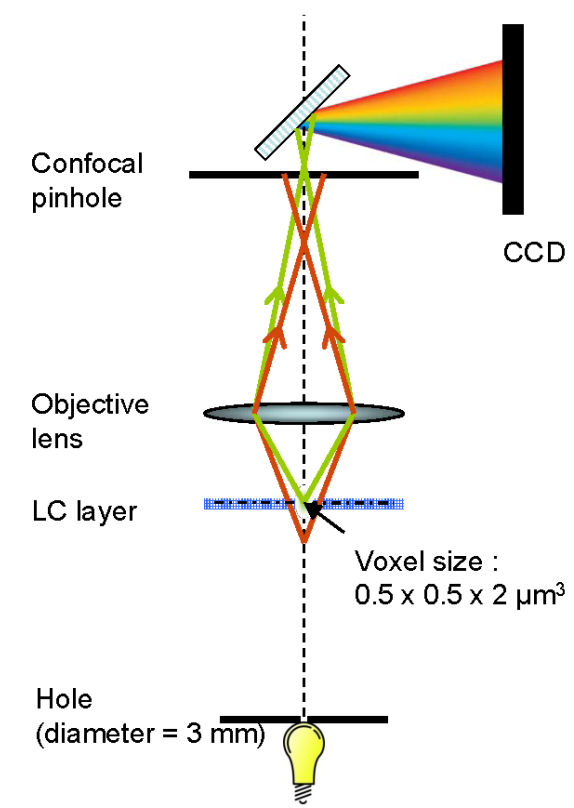

Fig. 2 Experimental setup. An original investigation technique combines confocal microscopy and spectrophotometry (XploRA ${ }^{\mathrm{TM}}$ from Horiba). A $3 \mathrm{~mm}$ diameter circular hole is placed above the halogen light source at a distance of $8 \mathrm{~cm}$ below the layer in order to approximate a point source. The back projection of the confocal pinhole in 3D defines the unit volume $\left(0.5 \times 0.5 \times 2 \mu \mathrm{m}^{3}\right)$ analysed for a fixed position. The scanning step was $0.5 \mu \mathrm{m}$ along the transverse $\mathrm{XY}$ direction and $4 \mu \mathrm{m}$ along the longitudinal $Z$ direction. For each position, the spectrum in the visible range of the transmitted light is recorded. 
The transmittance spectrum of the polygon is recorded on every point of the area. Maps of optical transmission are then rebuilt for different spectral ranges. The light enters the layer from the substrate side. Three distinct spectral bands in the visible range are chosen to fully describe the wavelength-tunable light shaping properties of the optical film (Fig. 3): (i) the reflection band as measured from the top side of the layer, or Bragg band; (ii) a high transmission band, which was called the band edge; (iii) the red band, far from the Bragg regime. The Bragg band and the band edge undergo a blue-shift (Fig. 4) when the annealing time increases, which is the direct consequence of the time-controlled changes to the helical axis orientation from the top of the layer to the bottom of the CLC structure ${ }^{23}$.

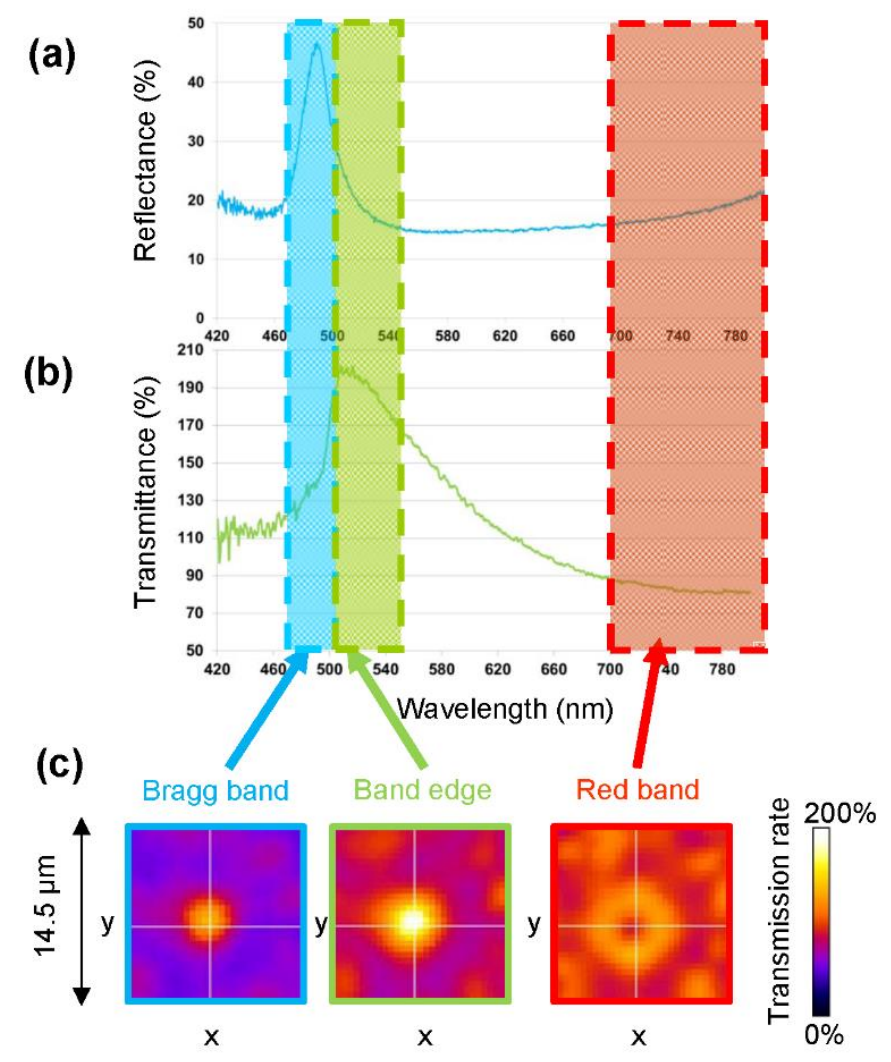

Fig. 3 Defining the three spectral bands to demonstrate the wavelength-tunability of light-shaping. The light shaping property of a single polygon as a function of the wavelength is investigated in three different spectral bands which are of interest for their optical properties. (a) Reflectance and (b) 
transmittance spectra in the Bragg band, band edge and red band. (c) XY maps. The white crosses on the maps mark the coordinates of the locus where the spectra in $a$ and $b$ were recorded. The pixel size is $0.5 \times 0.5 \mu \mathrm{m}^{2}$. The layer was annealed at $140^{\circ} \mathrm{C}$ for 5 hours.

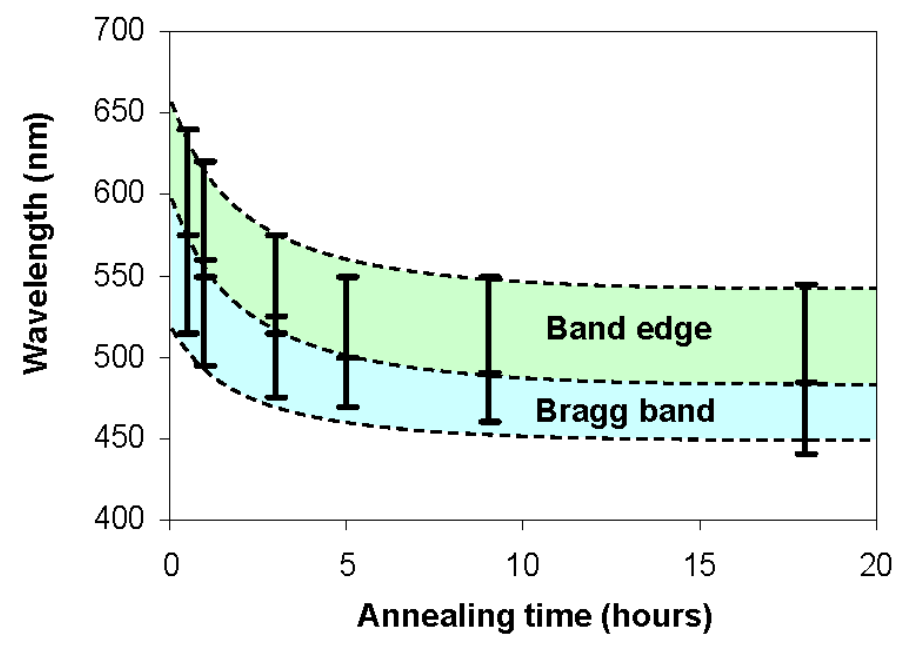

Fig. 4 Blue shift of the Bragg band and the band edge during the annealing process. Changes of the helix axis orientation from the air-material interface lead to a blue shift of the reflected colour. This is because the mean reflection wavelength $\lambda_{0}$ is directly related to the value of the angle $\theta$ between the propagation direction and the helix axis according to Bragg's law $\lambda_{0}=n p \cos \theta$. $n$, the average of refractive indices, and $p$, the helical pitch, remain constant during this process ${ }^{23}$. The vertical bars indicate the spectral ranges used to define the Bragg band and the band edge as a function of the annealing time. After 18 hours, the colour does not change anymore and the system is considered to have reached equilibrium.

Fig. 5 shows the maps of the transmitted light through the layer after 5 hours of annealing at $140^{\circ} \mathrm{C}$ and quenching at RT. The probe area is centred on a single polygon with a diameter of $11.5 \pm 1 \mu \mathrm{m}$. The colour scale represents the transmission per area unit (the baseline is recorded with the glass substrate only). Since the light is differently concentrated on the polygon surface, the 
transmission scale can thus reach values above $100 \%$. The optical behaviour of a polygon in the three spectral bands is reported for three dimensions: $X Y$ view in the film plane (top), and $X Z$ and $Y Z$ crosssectional views (bottom). The $X Y$ maps correspond to the $Z$ height for which the transmittance is maximum. These $X Y$ maps define the focus plane, which is marked in cross-sections with a black dotted line. In the Bragg band and band edge, the focus planes are localized beyond the optical layer, which means that the focal length is negative (diverging lens). The focus point is thus a virtual image of the point source (which is a circular hole with a diameter of $3 \mathrm{~mm}$ at a distance of $8 \mathrm{~cm}$ below the layer) formed by the polygon. The $X Z$ and $Y Z$ vertical cross-sections are built from a stack of $X Y$ images recorded at different $Z$ heights with a step equal to $4 \mu \mathrm{m}$. These cross-sections are thus not a temporal representation of propagation, for which the light before the layer would be the incident plane wave. This is why the distribution of the transmitted intensity below the layer appears to be non-homogenous. From these cross-section views, it is clear that the focus spot has a cylindrical symmetry. In the Bragg band (resp. band edge), the radial intensity distribution can be fitted with a Gaussian function; the maximum intensity reaches $290 \%$ (resp. $360 \%$ ) of the incident light and the full width at half maximum is equal to $2.5 \mu \mathrm{m}$ (resp. $2.8 \mu \mathrm{m}$ ). The full profiles are given in Fig. 6 . The background intensity level is about $50 \%$ (resp. 80\%) and quite homogeneous. The significant difference in transmission in the Bragg band and the band edge is due to the light reflection of the layer (Supplementary Fig. S2). In the red band, the beam shaping is very different: the focus plane is located in the layer and the maximum light intensity is distributed in a bright donut-like ring that surrounds the polygon centre. The $X Z$ and $Y Z$ maps show that the light is guided through the sample along a hollow cylinder with a mean diameter of about $6.0 \mu \mathrm{m}$. Since the transverse profile of the light does not undergo any variation along the entire layer thickness, the material acts as a propagation mode selector. The polarization states of this mode are radially distributed as demonstrated by the analysis of polarization in the XY planes (Supplementary Fig. S3). Such cylindrical vector beams have attracted great interest in recent years due to their unique properties, 
which are exploited in a variety of applications, like super resolving microscopy based on fluorescence depletion process ${ }^{36}$, laser trapping ${ }^{37}$ or tighter focusing ${ }^{38}$.

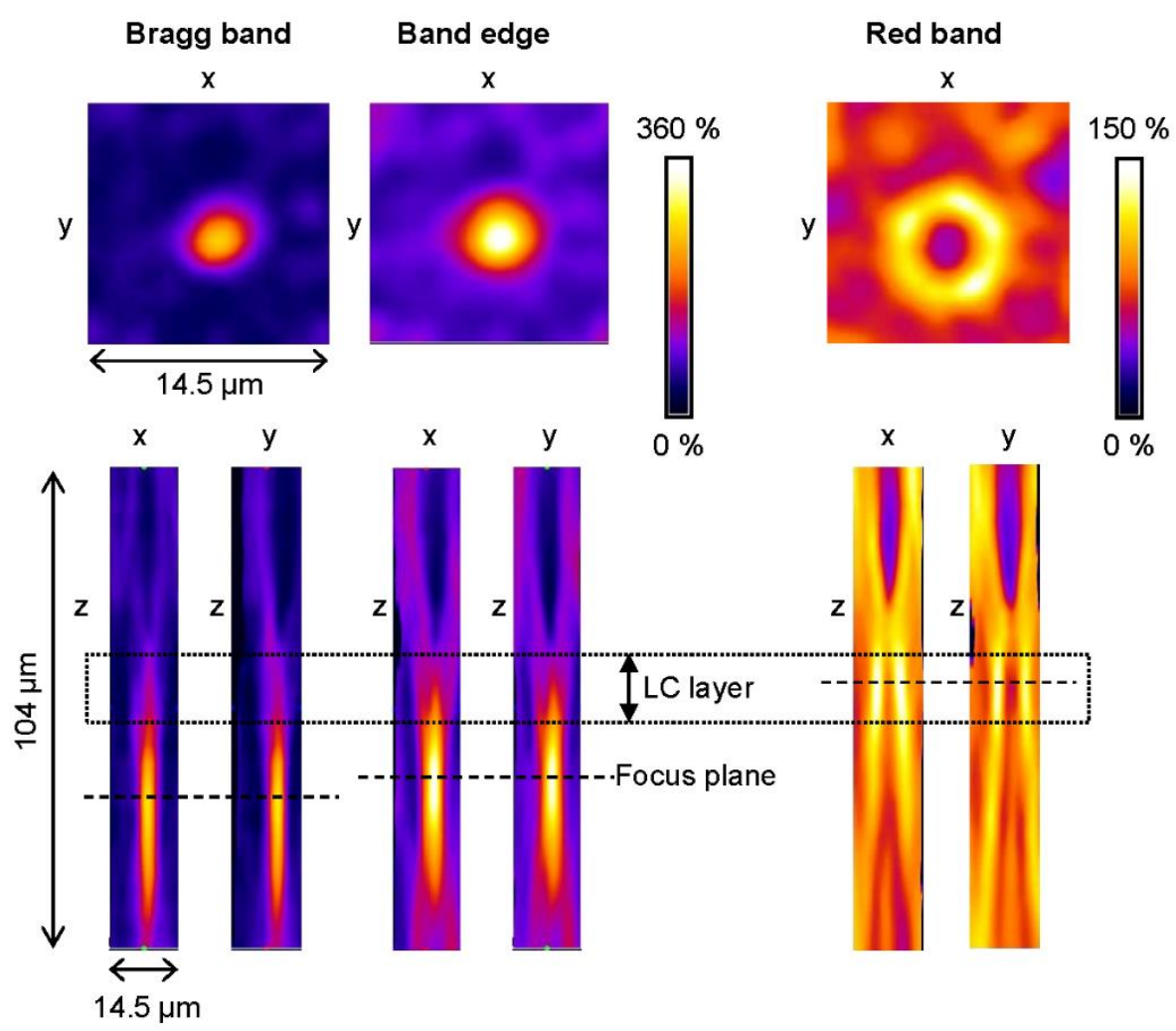

Fig. 5 Wavelength-dependence of the focus locus. Mapping of the light transmitted through the optical film that was annealed at $140^{\circ} \mathrm{C}$ for 5 hours in the film $\mathrm{XY}$ plane (top) and in the $\mathrm{XZ}$ and $\mathrm{YZ}$ cross-sections (bottom). Three spectral bands of interest were chosen as follows: Bragg band (470$500 \mathrm{~nm})$, band edge $(500-550 \mathrm{~nm})$ and red band $(700-800 \mathrm{~nm})$. The colour scale refers to the percentage of transmission per area unit. The scale assigned to the red band is different than those related to the Bragg band and band edge to facilitate the representation. The position of the film is marked in the cross-section maps. The focus plane is marked with a dotted line. XY maps are presented for the $\mathrm{Z}$ focus plane corresponding to the local maximum of transmission. $\mathrm{XZ}$ and $\mathrm{YZ}$ maps are built by stacking the $X Y$ maps obtained at different $Z$ foci. The shape of the light propagation depends on the wavelength range; either inside a tunable-transmission lens with a cylindrical symmetry in the Bragg band and the band edge, or inside a donut-like ring in the red band. 


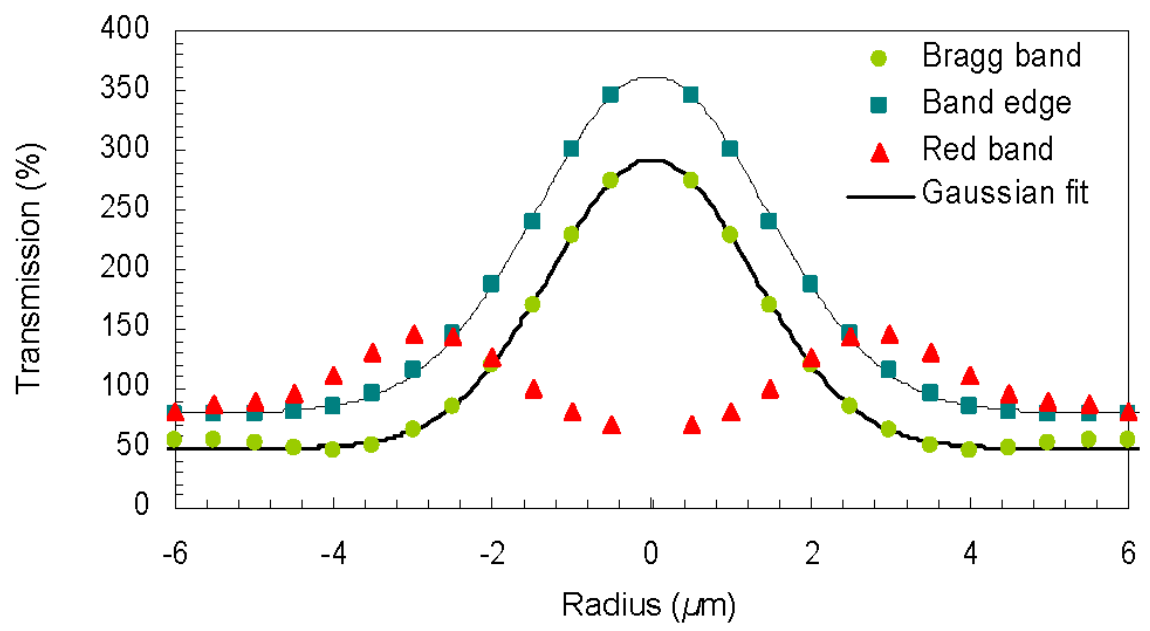

Fig. 6 Radial profiles at the focal plane. Plots of normalized integrated transmission around concentric circles as a function of distance from the optical axis. The transmission rate at any given distance from the centre represents the sum of the pixel values of the $X Y$ map around a circle. This circle is centred on the optical axis, and its radius is the distance from that axis. The integrated transmission rate is divided by the number of pixels in the circle, yielding normalized comparable values. Dots represent experimental values for the Bragg band (circles), the band edge (squares) and the red band (triangle). The solid lines are Gaussian fits.

Fig. 7 demonstrates the versatility of the polygon design as a simple function of annealing time by displaying the $X Y$ spatial distribution of the light intensity at the focus plane. The Bragg band is blueshifted from 515-575 nm (30 minutes annealing time) to 440-485 nm (18 hour annealing time) (Fig. 4) due to the propagation of the tilt of the helical axis into the bulk material. Inside the Bragg band for the material annealed for 30 minutes, the transmitted light is quite homogeneous and a weak donut profile begins to emerge. At 1 hour, a central bright spot takes shape. The focusing effect is thus more efficient after 1 hour and it increases with time. For a longer time (typically after 10 hours), the cholesteric structure does not evolve further and the optical effect saturates. In the band edge, the scenario is quite similar, except the central spot nucleates later ( 3 hours) and the 
transmission rate is higher (due to the reflection which is less important than in the Bragg band). Into the red band, the light is shaped inside the material into a donut even after long annealing times.

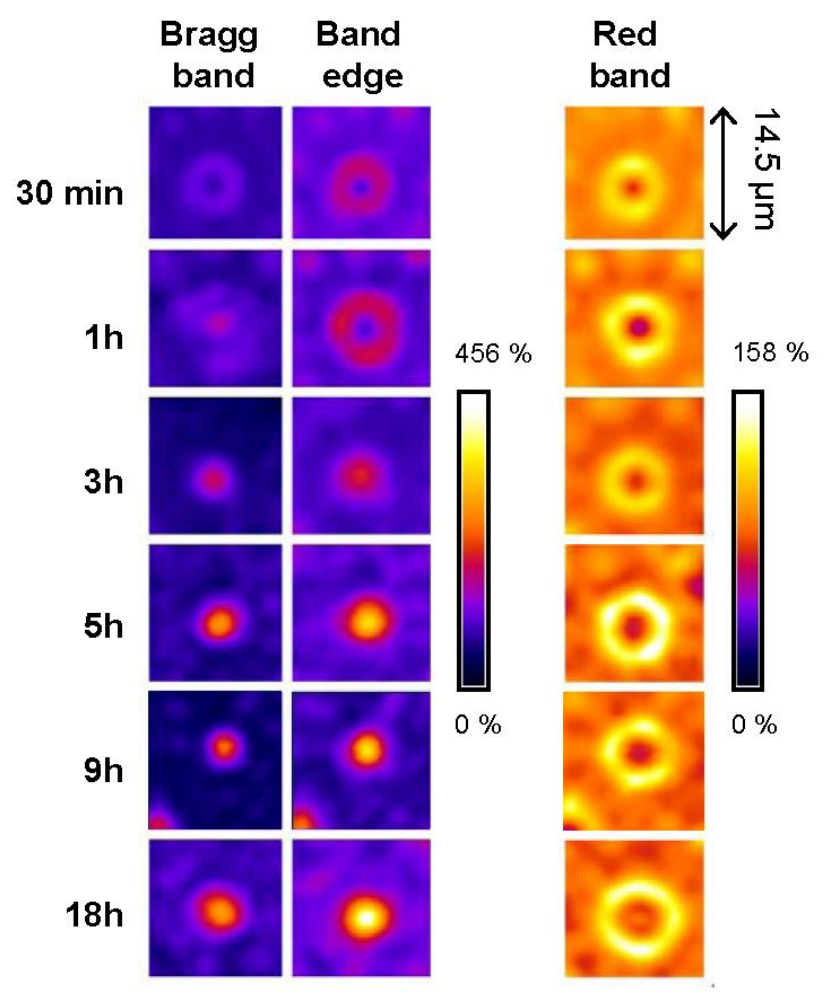

Fig. 7 Design of light shaping. Light shaping is achieved by designing the polygon structure. Elegantly, this structure only depends on the annealing time of the optical layer. The mapping of the light intensity at the focus plane is displayed for annealing times ranging from 30 minutes to 18 hours for the three basic wavelength bands.

Since the open film spontaneously develops polygonal units after annealing, self-organized arrays of microlenses are easy to produce (Supplementary Fig. 8). The homogeneity of the light shaping between neighbouring polygons is remarkable since the polygons have the same profile in the same $Z$ plane. A Fourier transformation of a set of adjacent polygons on a surface equal to $40 \times 40 \mu \mathrm{m}^{2}$ shows the spectral signature of a regular hexagonal lattice (Fig. $8 \mathrm{~d}$ ). The technological production of microlenses in a regular pattern with a perfectly uniform size is out of the scope of the present work. 
However, although the formation of the polygonal texture results from a spontaneous (uncontrolled) self-assembly mechanism, this result indicates that it is easy to find a set of microlenses that are periodically self-organized. The layer is not composed of a collection of shaped items on a specific substrate but is the result of a tailoring of the structure within the single layer. The fact that the optical layer is a 3D-organized film in a liquid-crystalline state ensures the full continuity of the global architecture. This unique property minimizes undesired diffraction due to the lens edges and optimizes the fill factor. When the light distribution in the focus plane is a central spot (in the Bragg band and band edge), a microchannel acts as a microlens and an optical image of a distant object can be recorded (Fig. 9). The orientation of the collected image versus the position of the objectrepresented by a usual " $\mathrm{F}$ "-implies that the image is virtual. This is in agreement with the position of the focus plane located beyond the sample. The linear dependence of the focus length on the polygon diameter was determined (Fig. 9 d). In the case depicted here, the image height is about $6 \mu \mathrm{m}$. At this scale, the diffraction limit avoids the formation of images with sharp edges. A simple demonstration which consists of splitting the three RGB channels of the 3-CCD camera used to record the image (Fig. 9 e) highlights the strong wavelength selectivity of the microlens. By virtue of its structure, the optical layer combines the properties of a microlens and a colour filter. As a selfassembled material, this new type of lens can find use in interesting applications in all-integrated colour-sensitive devices like CMOS image sensors. Indeed, most of current devices are composed of a substrate, a manufactured array of microlenses and a colour filter layer. 


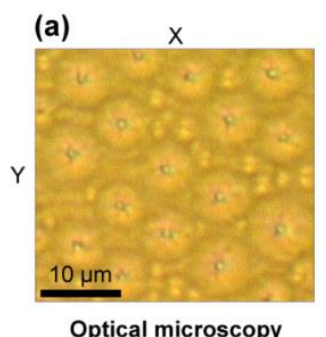

Optical microscopy

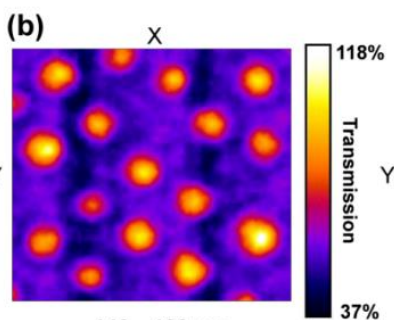

$440-460 \mathrm{~nm}$

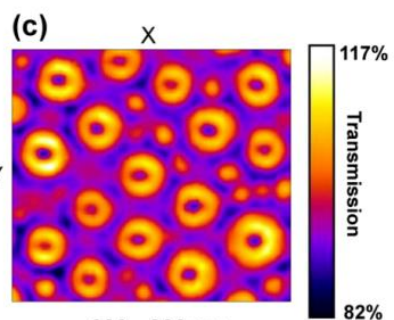

$600-800 \mathrm{~nm}$

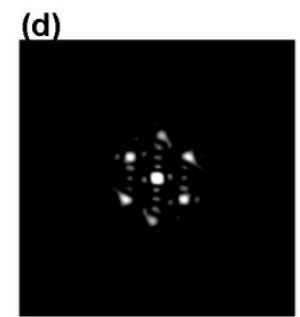

Fourier transform

Fig. 8 Self-organized array of microlenses. (a) Optical micrograph in transmission mode (unpolarised light). The layer was annealed at $140^{\circ} \mathrm{C}$ for 13 hours. (b) Imaging of the array of focus points by confocal microscopy in the Bragg band. (c) Imaging of the array of donut-like foci by confocal microscopy in the red band. (d) 2D spatial Fourier transform of image b. The 6 bright spots regularly arranged around a central point are testimony to the existence of a hexagonal pattern in the selfarrangement of polygons in that region. The a-c images were recorded at the same $Z$ height. The scanning step of the $\mathrm{XY}$ maps is $0.5 \mu \mathrm{m} \times 0.5 \mu \mathrm{m}$. The polygon size, defined as the centre-to-centre distance, depends on the film thickness. In the present case, the polygons are statistically arranged. No specific effort was made here to force the microlenses to nucleate at periodic sites and to grow with the same lateral sizes (this objective is out of the scope of the present work). The lateral size of the polygons has a large distribution and is thickness dependent. Smaller size polygons might be fabricated by decreasing the layer thickness, but to the detriment of the light intensity transmitted through the microlenses. 
(a)

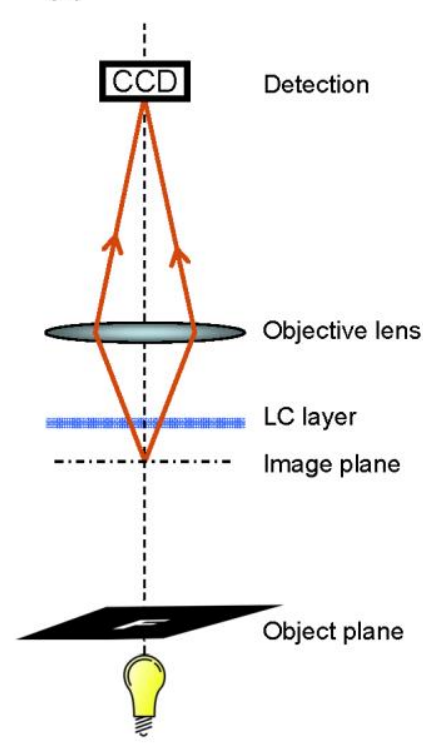

(b)

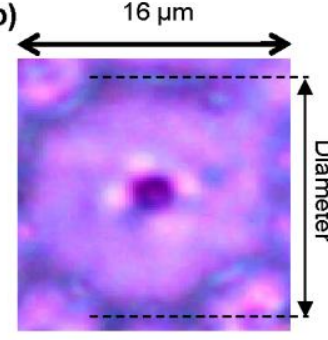

On the top of LC layer (c)

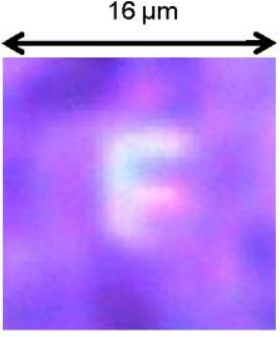

On the image plane (d) Diameter $(\mu \mathrm{m})$

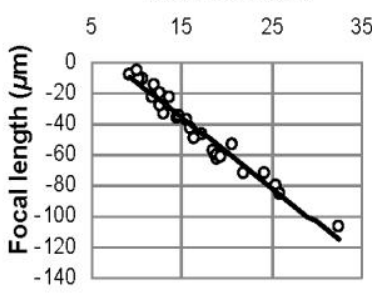

(e) $\mathbf{R}$

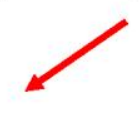

B
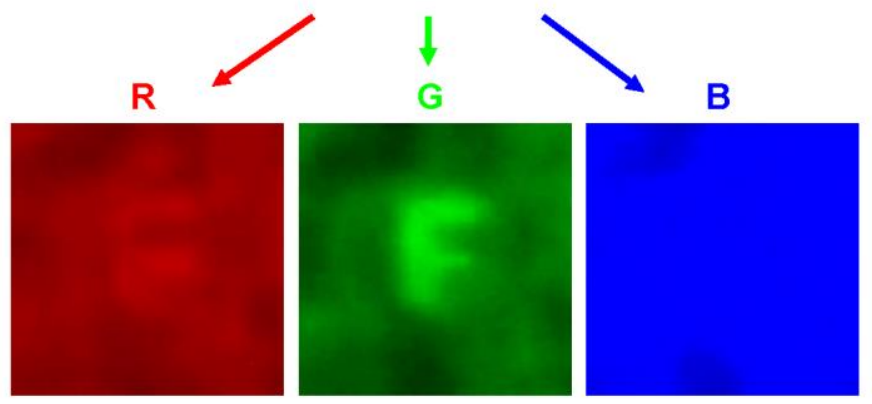

Fig. 9 Wavelength-selective imaging. (a) Experimental microscope configuration used to record an optical image of the traditional " $F$ " object through (b) a single microlens. The height of object " $F$ " is 2 $\mathrm{cm}$ and is located at an $8 \mathrm{~cm}$ distance beyond the layer. (c) The virtual image located beyond the layer recorded onto a CCD camera by way of an objective lens. (d) Variation of the focal length of a microlens as a function of the polygon diameter. (e) Evidence of the intrinsic colour filter property of the microlens; the three images correspond to the 8-bit RGB channels of the 24-bit colour image depicted in $c$.

\section{Numerical simulations and discussion}

What is basically at the origin of the light shaping and the spectral selectivity of a polygon? A classical glassy lens transmits and refracts light. Fresnel lenses and gradient index (GRIN) lenses are known for focusing light. The physical processes involved in the light propagation along a microlens cannot be explained by any physical mechanism at the origin of Fresnel and GRIN lenses. First, the wavelength selectivity is due to the peculiar morphology of the Bragg grating into the layer. The central 
wavelength of the Bragg reflection in a CLC is defined by $\lambda_{0}=n p \cos \theta$, where $n$ is the mean refractive index, $p$ is the pitch value and $\theta$ is the angle between the incident light and the helix axis. In the TEM image of a cross-section, as viewed in Fig. 10 a, the number of molecular $2 \pi$-rotations along the central axis (dashed line A) is lower than along a line on the left side (e.g. dashed line B) or right side. Additionally, the mean value of the angle $\theta$ is higher along line $B$. The association of these two structural properties leads to a transverse modulation of the central reflection wavelength $\lambda_{0}$. In other words, $\lambda_{0}$ is higher in the middle of a polygon and gradually decreases around it. In order to demonstrate the basic role of this particular Bragg grating onto the wavelength-tunability of a microlens, FDTD (finite difference time domain) numerical simulations were performed by only taking into account the Bragg modulation of the index (Details in Supplementary Information). Fig. $10 \mathrm{~b}$ shows the refractive index mapping used to perform the simulations. This mapping is mathematically generated to fit with the features (pitch, $\theta$ distribution, $\Delta \mathrm{n}$, image resolution) of the experimental stripe texture as seen in Fig. 10 a. Fig. 10 c (resp. d) depicts the electric-field energy density in the layer as numerically calculated when a monochromatic plane wave with a wavelength equal to $\lambda=480 \mathrm{~nm}$ (resp. $600 \mathrm{~nm}$ ) propagates into the layer by entering from below. These images are not directly comparable to the experimental $X Z$ maps because they depict different quantities (transmission vs. electric-field energy density). Nevertheless, the selective shaping of the propagative wave is clearly visible and confirms the role of the nested arc cholesteric structure. In particular, the waveguide behaviour of the microlens far from the Bragg band is reproduced. 
(a) Experimental

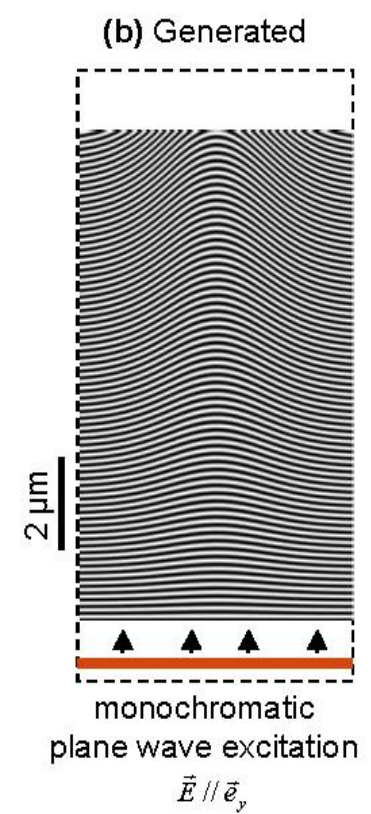

(c) $\lambda=480 \mathrm{~nm}$

(d) $\lambda=600 \mathrm{~nm}$
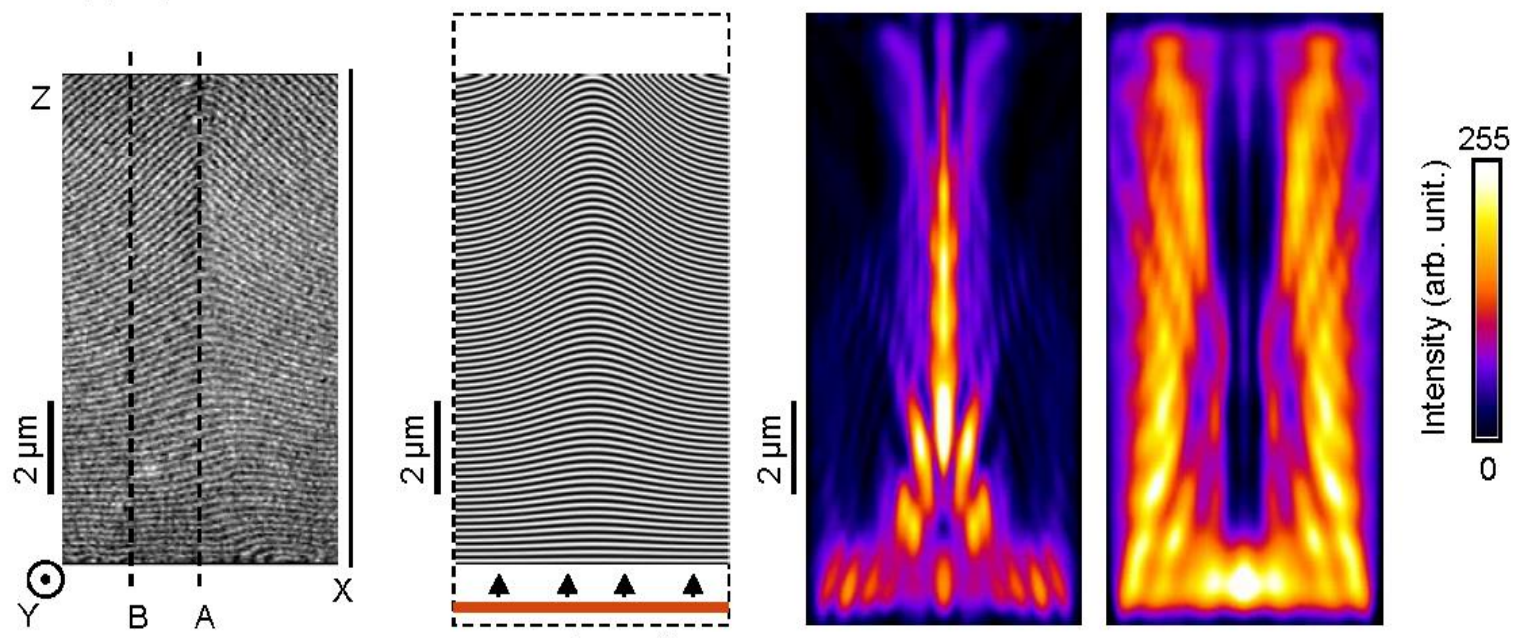

Fig. 10 Simulated light shaping through the chiral structure of a single microlens. (a) TEM crosssection image of the nested arc structure with an annealing time of 13 hours. The vertical dashed line $A$ is the optical axis of the microlens and it passes by the centre of the polygon on the surface. As the contrast is related to the orientation of the molecules, the periodicity corresponds to half a pitch. (b) Generated index mapping used for numerical simulations. The physical parameters were given by the experimental data as follows: mean index $n=1.57$, birefringence $\Delta n=0.3$, pitch $p=350 \mathrm{~nm}$. The index value varies from 1.42 in the black zones to 1.72 in the white zones. (c) and (d) Numerically calculated energy density envelop of the electric field during the propagation of an incoming monochromatic plane wave through the material depicted in $b$. The shaping of the transmitted wave is strongly dependent to the wavelength. In the Bragg band (c, $\lambda=480 \mathrm{~nm}$ ), inside the material the flux is confined along the optical axis and most of the incoming light is reflected. Outside of the Bragg band $(d, \lambda=600 \mathrm{~nm})$, a donut transverse mode is selected during the propagation through the medium, as observed in experiments. 
With respect to the physical origin of the lens effect, i.e. the ability to form an image from a distant object, a gradient index may be the cause of this effect. The variations of the molecular director field into the structure cause a gradient in the refractive index that cannot be neglected. However, the effective index should be higher in the centre of a polygon (where the molecules are planar, i.e. $\theta=0$ ) than around the periphery of the polygon (where $\theta>0$ ), so the lens should be convergent. The diverging property of the lens is maximized around the band edge as illustrated by the peak in the transmission spectrum in Fig. 3. It is known that, in the vicinity of the band edge, the group velocity drastically decreases ${ }^{39}$ concomitantly with a large increase in the optical path. The position of the band edge has the same transverse modulation in the frequency space as the one of $\lambda_{0}$ as described before. As a consequence, the optical path can be shorter for wave propagating along the optical axis than for wave propagating around it. The microchannel thus acts as a divergent lens. Such applications of anomalous indexes of refraction in photonic bandgap materials were theoretically foreseen two decades ago ${ }^{39}$. The specific distribution of the CLC Bragg grating provides a continuous transverse modulation of $\lambda_{0}$ without any defect and is responsible for both the light shaping effect and its wavelength selectivity.

\section{Conclusions}

In summary, (i) in the polygonal cholesteric texture, a polygon acts as a microlens or -channel that moulds the light. This effect is wavelength-tunable. (ii) We demonstrate the control of the shape design of the focus locus by controlling the annealing time. Both results ( $i$ and ii) lead to the fabrication of all organic microlenses as a simple way to modulate the optical and geometrical characteristics of the polygons. The total self-assembly method for fabrication of an array of these microlenses does not require any surface-modified substrate, which may be a flat or curved glass plate or a sheet of rollable plastic. (iii) The light shaping is basically controlled by the intrinsic cholesteric structure of the lens. (iv) The wavelength selectivity of the focus shape comes from the 
wavelength dependence of the Bragg reflection on the conditions of the variable angle between the normal to the film plane and the helical axis. This new type of optical device may be named a "Bragg lens". It encompasses a multitude of tunability properties and the specific interaction between light and the CLC layer leads to wavelength-selective shape of the focus locus-either a spot or donut. The present material addresses the domain of integrated photonics applications which require optical elements that are cheap, highly tunable and can operate in different directions with ultrasmall mode volumes. These versatile and easy-to-produce polygons may be used as directional light sources in soft-matter photonic circuits in the future.

\section{Acknowledgements}

The authors thank Dr. E. Hanelt from Wacker-Chemie GmbH (Munich, Germany) for providing them with oligomers and constant support, and Dr. A. Zwick (CEMES-CNRS, Toulouse) for his technical assistance with Xplora.

\section{References}

1. N. Engheta, Science, 2011, 334, 317-318.

2. C. Battaglia, J. Escarré, K. Söderström, M. Charrière, M. Despeisse, F.-J. Haug, and C. Ballif, Nat Photon, 2011, 5, 535-538.

3. L. Novotny and N. van Hulst, Nat Photon, 2011, 5, 83-90.

4. X. Ni, N. K. Emani, A. V. Kildishev, A. Boltasseva, and V. M. Shalaev, Science, 2012, 335, 427-427.

5. Y. Yang, L. K. Chin, J. M. Tsai, D. P. Tsai, N. I. Zheludev, and A. Q. Liu, Lab on a Chip, 2012, 12, 3785.

6. Y. C. Seow, S. P. Lim, and H. P. Lee, Lab on a Chip, 2012, 12, 3810.

7. W. Song and D. Psaltis, Lab on a Chip, 2013, 13, 2708. 
8. C. W. Hsu, B. Zhen, J. Lee, S.-L. Chua, S. G. Johnson, J. D. Joannopoulos, and M. Soljačić, Nature, 2013, 499, 188-191.

9. J.-H. Park, C. Park, H. Yu, J. Park, S. Han, J. Shin, S. H. Ko, K. T. Nam, Y.-H. Cho, and Y. Park, Nat Photon, 2013, 7, 454-458.

10. T. Miyashita, Jpn. J. Appl. Phys., 2007, 46, 5391.

11. P. Nussbaum, R. Völkel, H. P. Herzig, M. Eisner, and S. Haselbeck, Pure Appl. Opt., 1997, 6, 617.

12. M.-H. Wu and G. M. Whitesides, Adv. Mater., 2002, 14, 1502-1506.

13. D. L. MacFarlane, V. Narayan, J. A. Tatum, W. R. Cox, T. Chen, and D. J. Hayes, IEEE Photonics Technology Letters, 1994, 6, 1112-1114.

14. F. Chen, H. Liu, Q. Yang, X. Wang, C. Hou, H. Bian, W. Liang, J. Si, and X. Hou, Opt. Express, 2010, 18, 20334-20343.

15. D. Daly, R. F. Stevens, M. C. Hutley, and N. Davies, Meas. Sci. Technol., 1990, 1, 759.

16. X. Li, Y. Ding, J. Shao, H. Tian, and H. Liu, Adv. Mater., 2012, 24, OP90-OP90.

17. M. Fujita, S. Takahashi, Y. Tanaka, T. Asano, and S. Noda, Science, 2005, 308, 1296-1298.

18. K. Ishizaki and S. Noda, Nature, 2009, 460, 367-370.

19. C. Xiao, N. Fujita, K. Miyasaka, Y. Sakamoto, and O. Terasaki, Nature, 2012, 487, 349-353.

20. S. P. Palto, L. M. Blinov, M. I. Barnik, V. V. Lazarev, B. A. Umanskii, and N. M. Shtykov, Crystallogr. Rep., 2011, 56, 622-649.

21. D. C. Zografopoulos, R. Asquini, E. E. Kriezis, A. d' Alessandro, and R. Beccherelli, Lab on a Chip, 2012, 12, 3598.

22. F.-H. Kreuzer, N. Häberle, H. Leigeber, R. Maurer, J. Stohrer, and J. Weis, in Organosilicon Chemistry Set, eds. N. Auner and J. Weis, Wiley-VCH Verlag GmbH, 2005, pp. 566-586.

23. G. Agez, R. Bitar, and M. Mitov, Soft Matter, 2011, 7, 2841.

24. Y. Bouligand, Journal de Physique, 1972, 33, 715-736.

25. Y. Bouligand, Tissue and Cell, 1972, 4, 189-217.

26. M. Mitov and P. Sixou, Journal de Physique II, 1992, 2, 1659-1670. 
27. M. Mitov and P. Sixou, Molecular Crystals and Liquid Crystals Science and Technology. Section A. Molecular Crystals and Liquid Crystals, 1993, 231, 11-28.

28. R. Bitar, G. Agez, and M. Mitov, Soft Matter, 2011, 7, 8198.

29. K. Lee, W. Wagermaier, A. Masic, K. P. Kommareddy, M. Bennet, I. Manjubala, S.-W. Lee, S. B. Park, H. Coelfen, and P. Fratzl, Nat. Commun., 2012, 3, 725.

30. L. P. Biró and J. P. Vigneron, Laser \& Photon. Rev., 2011, 5, 27-51.

31. J. Aizenberg, A. Tkachenko, S. Weiner, L. Addadi, and G. Hendler, Nature, 2001, 412, 819-822.

32. A. Pace, Science, 1972, 176, 678-680.

33. V. Sharma, M. Crne, J. O. Park, and M. Srinivasarao, Science, 2009, 325, 449-451.

34. K. Allahverdyan, T. Galstian, A. Gevorgyan, and R. Hakobyan, Optics and Photonics Journal, 2013, 03, 17-22.

35. A. Saupe, Molecular Crystals and Liquid Crystals, 1973, 21, 211-238.

36. B. Neupane, F. Chen, W. Sun, D. T. Chiu, and G. Wang, Review of Scientific Instruments, 2013, 84, 043701.

37. H. He, M. E. J. Friese, N. R. Heckenberg, and H. Rubinsztein-Dunlop, Phys. Rev. Lett., 1995, 75, $826-829$.

38. Q. Zhan, Adv. Opt. Photon., 2009, 1, 1-57.

39. J. P. Dowling, M. Scalora, M. J. Bloemer, and C. M. Bowden, Journal of Applied Physics, 1994, 75, 1896-1899. 


\section{SUPPLEMENTARY INFORMATION}

\section{Wavelength-tunable light shaping with cholesteric liquid crystal microlenses}

By Chloé Bayon, Gonzague Agez and Michel Mitov*

Centre d'Elaboration de Matériaux et d'Etudes Structurales (CEMES), CNRS, University Paul-Sabatier, 29 rue J. Marvig, 31055 Toulouse cedex 4, France.

*E-mail : mitov@cemes.fr

This section contains: Details on numerical simulations and Supplementary figures S1-S3.

\section{Details on numerical simulations}

In order to numerically calculate the propagation of light across the material, numerical replica were generated. Meaning that the pitch value, the distribution of the orientation of the helical axis, the spatial resolution and the dielectric birefringence match the experimental features. Simulations were performed by using the finite-difference time-domain (FDTD) method of the Meep ${ }^{\mathrm{s}}$ software package. This electromagnetic simulation software uses Maxwell equations and we let them run over time within some finite computational region. The computational grid resolution is 60 pixels per $\mu \mathrm{m}$. The $2 \mathrm{D}$ box size is 800 pixels along the direction of light propagation and 360 pixels along the transverse direction. We imposed absorbing boundary conditions (the present approach utilizes a setup where the computational cell is surrounded with a medium that absorbs light without any reflection). We set the average refractive index $n$ at 1.57 and the birefringence $\Delta n$ at 0.3 . The incoming light is a continuous plane wave source proportional to $\exp (-i \omega t)$ with $\omega=2 \pi c / \lambda$, where 
$\lambda$ is the wavelength and $c$ is the speed of light in a vacuum. The polarization is linear and orthogonal to the XZ plane. After a transient regime, 300 images of the electric-field energy density $E^{*} . D / 2$ are recorded during 100 periods. The energy density from both the forward and the backward (due to Bragg reflection) fields are recorded. This quantity oscillates with a pulsation equal to $2 \omega$. The envelopes are then extracted with a pass band filter centred at $2 \omega$ in the Fourier space. The pictures in Fig. $10 \mathrm{c}$ and $\mathrm{d}$ are time averaged over the 100 periods (300 images). The colour scale is proportional to the intensity.

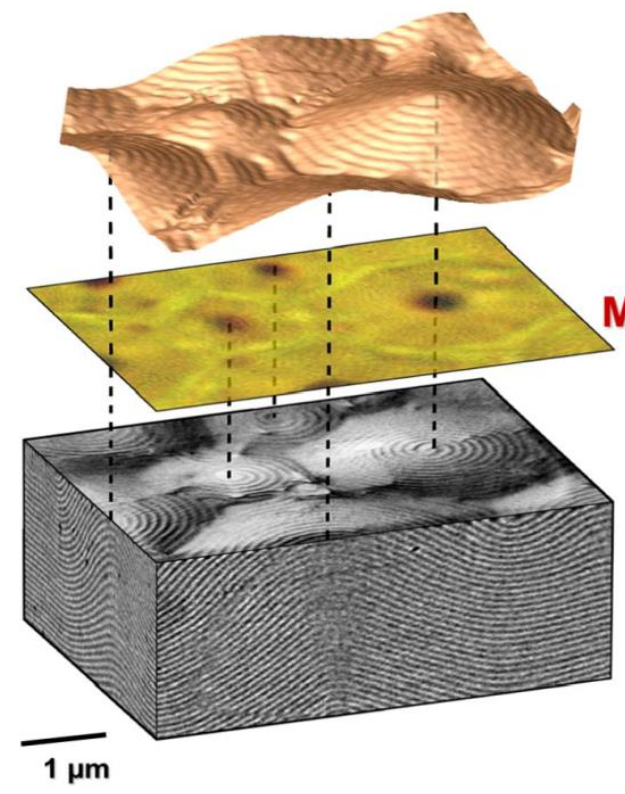

(a)

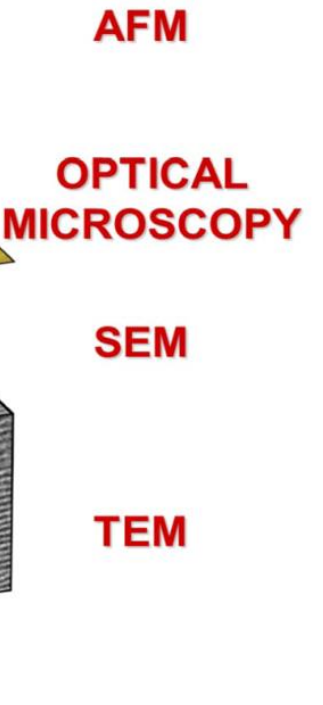

(b)

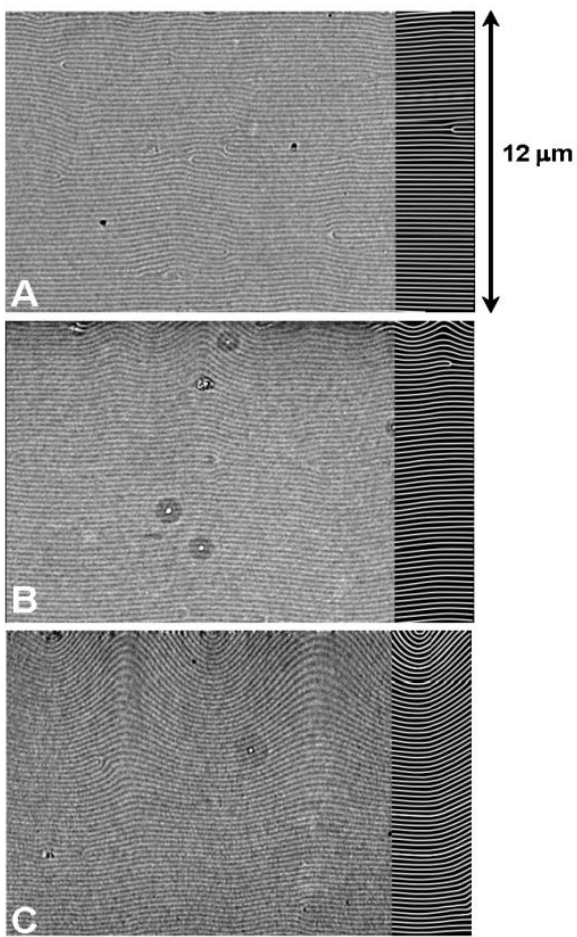

Fig. S1 Structure of the optical layer. The open cholesteric-liquid-crystalline film was annealed at $140^{\circ} \mathrm{C}$ for 13 hours, and then quenched at room temperature. It exhibited hybrid anchoring after annealing; meaning the preferential molecular orientation is planar on the glass substrate and it is homeotropic (i.e. perpendicular) at the air interface. This hybrid anchoring leads to a specific cellular texture which is called the polygonal texture ${ }^{24,35}$. (a) 3D structure of the polygonal texture according to a combination of the following microscopy methods: atomic force microscopy (AFM), optical 
microscopy (OM), scanning electron microscopy (SEM) and transmission electron microscopy (TEM). The micrograph recorded with an optical microscope in transmission mode reveals a mosaic of polygons with various sizes. AFM imaging reveals the cone shape of polygons, which are the locus of double-spiral patterned lines. The connection between oblique lines in the TEM cross-sectional views and spiral patterns as seen in planar views by SEM is visible. Vertical dotted lines help visualize the coincidence between the central spots of cones from one image to the next. The relief of polygons results from the competition between the surface energy and the bulk free energy. The energy is lowered by transforming the free surface into a cone, thus reducing the bulk distortion energy at the cost of surface energy. The double-spiral structures correspond to the adaptation of the cholesteric structure to the relief and the conditions at the limits. A blue shift of the reflection band is caused by the interface-induced variation of the helical axis orientation, which progressively propagates further into the volume as the annealing time increases. (b) TEM images of cross sections of optical layers quenched at $140^{\circ} \mathrm{C}$ after various annealing times. The right part of each image was redrawn to facilitate the observation of texture changes. (A) No annealing; the fingerprint texture made of an array of periodic bright and dark lines is regularly parallel to both interfaces. The helix axis is perpendicular to the lines everywhere; the distance between two dark (or bright) lines represents the half pitch. (B) Annealing time = $1 \mathrm{~min}$; close to the air interface, the lines undulate, which already shows a change in the orientation of the helix axis. Further down, the lines remain parallel to the surfaces. (C) Annealing time $=13$ hours; the distortions invaded the bulk. Only a very thin material thickness close to the substrate exhibits quasi parallel lines. 


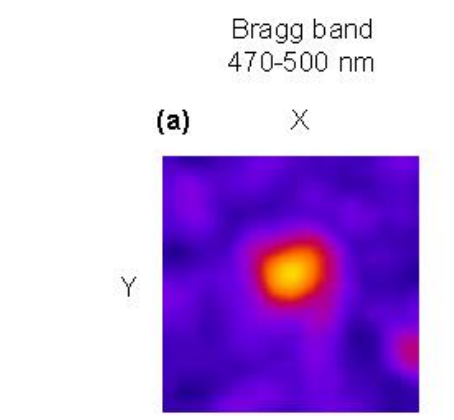

(c)

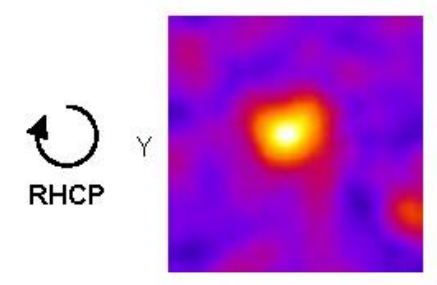

(e) $x$
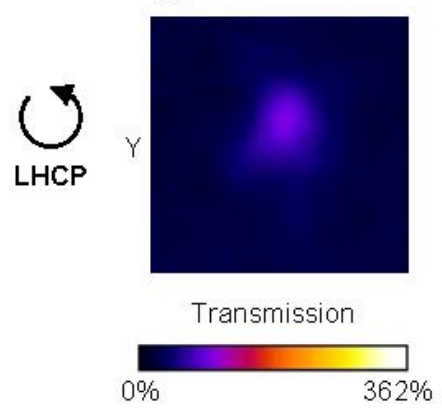

Band edge

500-550 nm

(b) $x$

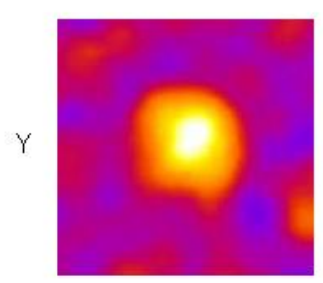

(d)

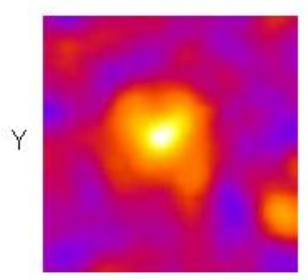

(f) $\times$

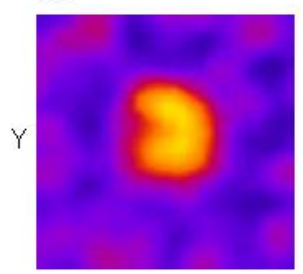

Transmission

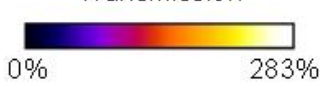

Fig. S2 Polarization dependent efficiency. Intensity profiles of the transmitted light when the incoming light is: (a-b) unpolarised; (c-d) right-handed circularly polarized (CP); (e-f) left-handed CP. $\mathrm{CP}$ light is obtained by combining a linear polarizer with a quarter-wave plate. The transmission rate inside the Bragg band is very weak (e) when the light has the same handedness (i.e. left) compared to the helical structure due to the light reflection and the polarization-selectivity rule in CLCs. When the light has the opposite handedness, the spot size is smaller than inside the band edge. When the incoming light is unpolarised, the maximum of efficiency is obtained in the band edge (b). 


\section{Red band $700-800 \mathrm{~nm}$}
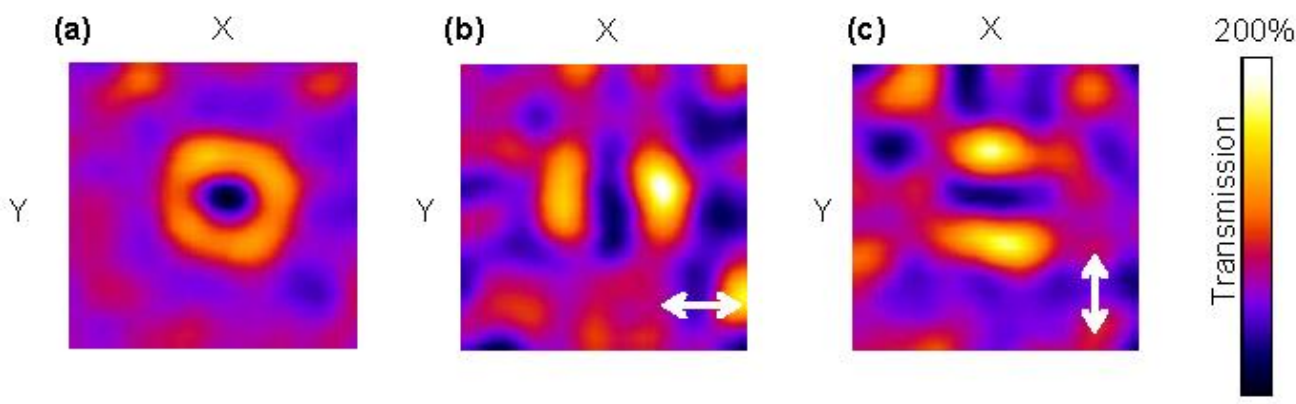

$50 \%$

Fig. S3 Evidence of the radial polarization state of the donut. Intensity profiles at the focal plane in the red band: (a) without analyser; (b) with an x-linear analyser; (c) with a y-linear analyser. The incoming light is unpolarised. The layer was annealed at $140^{\circ} \mathrm{C}$ for 5 hours. Along the donut ring the polarization is collinear to $k_{\perp}$, where $k_{\perp}$ is the orthogonal component of the Bragg grating wave vector.

S1. F. Ardavan et al., Comp. Phys. Comm., 2010, 181, 687-702. 